\title{
Management of bra in metastases from germ cell tumors: do we know what we are doing?
}

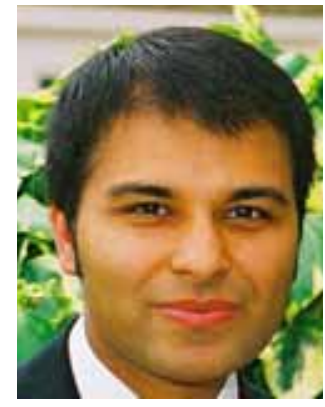

Sanjay Raj, Christine Parkinson, Michael Williams\& D anish M azhar ${ }^{\dagger}$

${ }^{\dagger}$ Author for correspondence Box 193, O ncology Centre, Addenbrookés H ospital, $\mathrm{H}$ ills Road, Cambridge CB2 0QQ, UK

Tel.: +44 122321 7020; Fax: +44 122327 4409; danish.mazhar@ addenbrookes.nhs.uk future, part of fso '....as many as $40 \%$ of germ cell

patients who die of progressive

tumor may have brain metastases

\section{at post mortem.'}

The introduction of cisplatin-based combination chemotherapy over 30 years ago has had a huge impact in the management of patients with advanced germ cell tumors (GCTs). Typically, patients who present with International Germ Cell Cancer Collaborative Group (IGCCC G ) good prognosis G CTs have a 5-year survival in excess of $90 \%$ [1]. By contrast, patients with poor prognosis, nonseminomatous (N S) G CTs have an estimated 5-year survival of $50 \%$. Included within this group are patients with CN S metastases. This is a rare phenomenon, occurring in only $1-2 \%$ of patients with germ cell cancers, though around $10 \%$ of patients with advanced GCTs present with brain metastases. H owever, as many as $40 \%$ of germ cell patients who die of progressive tumor may have brain metastases at post mortem [2]. Patients with brain metastases usually have widespread systemic disease, in particular, lung metastases. Even within the poor prognostic group, patients with brain metastases present a particularly challenging clinical problem, with long-term survival probably significantly lower than the 50\% quoted for the IGCCCG poor prognosis group. What is the optimal systemic chemotherapy regime, and indeed how reliable is the penetration of the blood-brain barrier of standard systemic cytotoxic drugs, is intrathecal chemotherapy indicated, what is the role of radiotherapy, and when is surgery indicated? These are some of the unanswered issues that compromise our understanding of this clinical situation and, hence, its management.

$O$ ne of the first points to make is that, given the relative rarity of the presentation, most of the available data is inevitably from small case series. Charing Cross Hospital published a series of nine men and one women with brain metastases at presentation from NSGCTS treated between 1977 and 1984 [3]. All the men had lung metastases, and in seven patients, the human chorionic gonadatrophin level was greater than 40,000 IU/I. Patients were treated with sequential combination chemotherapy with either POM B/ACE (cisplatin, vincristine, methotrexate, bleomycin, actinomycin, cyclophosphamide and etoposide) or EP/OMB (etoposide, cisplatin, vincristine, methotrexate and bleomycin), in which the methotrexate was given intravenously at a dose of $1 \mathrm{~g} / \mathrm{m}^{2}$ over $24 \mathrm{~h}$, and intrathecal methotrexate was given during courses not containing intravenous methotrexate. No patients received radiotherapy. O ne patient had surgery for residual computed tomography abnormalities, which proved to be gliosis. Eight patients were alive, off treatment with no active disease at 18 months. A total of two patients from the same study with primary intracranial N SGCTs were treated in a similar fashion. O ne patient died from enlargement of differentiated teratoma and the other was alive and in remission at 9 months. The authors concluded that chemotherapy should be the preferred treatment of primary or metastatic NSGCTs of the brain, and that only rarely will these patients benefit from surgery or radiotherapy.

The issue of which is the most effective chemotherapeutic treatment for patients with testicular cancer and brain metastases is controversial. The $C$ haring $C$ ross team has pioneered the use of POM B/ACE for metastatic GCTs and have published results suggesting superiority over 'standard' cisplatin-containing combination treatments in poor prognosis patients. H owever, these are retrospective data and comparisons are based on historical outcomes. There has never been a prospective randomized comparison. Intravenous methotrexate administered at $1 \mathrm{~g} / \mathrm{m}^{2}$ over $24 \mathrm{~h}$ can produce drug concentrations in the cerebrospinal fluid, which are considered to be within the cytocidal activity range of human tumors, and partial responses have been described to methotrexate alone [3]. Cisplatin, etoposide and bleomycin have been shown to penetrate through the blood-brain barrier in the presence of brain metastases [4,5]. These properties have been suggested as the explanation for the tumoricidal effect against CNS 
metastases observed with the Charing Cross regime. Curatively intended chemotherapy is necessary in all patients with brain metastases. Whether a 'CN S protocol' should be employed or whether conventional cisplatin-containing combination treatment should be given remains contentious. Reported long-term survival with the latter approach is as high as $45 \%$, although other treatment modalities are often employed in addition. Bokemeyer and colleagues summarized their long-term data from 44 patients with brain metastasis treated over a period from 1978- 1995 in $\mathrm{H}$ annover, Germany [6]. A total of 18 patients presented with brain metastases at primary diagnosis (group 1), four patients relapsed in the brain after previous favorable response to chemotherapy (group 2), and 22 (i.e., 50\%) developed brain metastases during or directly after chemotherapy (group 3). Chemotherapy generally consisted of cisplatin-based combination treatment such as PVB (cisplatin, vinblastine and bleomycin), BEP (bleomycin, etoposide and cisplatin) and PEI (cisplatin, etoposide and ifosfamide). No patient received intrathecal treatments. O verall, long-term survival was $23 \%$; however, the prognosis was significantly better for patients in groups 1 and 2 , with six out of 18 and three out of four patients alive at 2 years, respectively. Group 3 patients seemed to fair the worst, with only one out of $22(5 \%)$ surviving beyond 2 years, with a median survival in this group of 3 months. None of the patients treated with either chemotherapy $(n=6)$ or radiotherapy $(n=5)$ alone achieved long-term survival, while nine out of $28(32 \%)$ who received treatment with both modalities with or without surgery had sustained long-term survival (95\% Cl: $14.5-49.8 \% ; \quad p<0.03$ ). H owever, treatment modalities were not described according to group, making it virtually impossible to compare standardized outcomes of the different groups. In eight out of $26(31 \%)$ assessable cases, the cause of death was owing to progressive brain metastases, with all others dying owing to systemic tumor progression.

'The issue of which is the most effective chemothera peutic treatment for patients with testic ula rcancerand bra in meta sta ses is controversial.'

Fossa and colleagues published a multiinstitutional cohort of 139 patients with brain metastases from GCTs [7]. In total, 56 patients had brain metastases at diagnosis and all these patients received cisplatin-based combination chemotherapy, with supplementation with radiotherapy in 36 patients, and/or neurosurgery in ten cases. The 5-year overall survival (OS) was $45 \%$. N eurosurgery and the absence of extracerebral nonpulmonary metastases, but not the administration of radiotherapy, were found to be predictors of a better prognosis. A further 83 patients were identified who had brain metastases at relapse, a median of 9 months after initial chemotherapy. This group had a poor 5-year survival of $12 \%$. Radiotherapy was found to confer some benefit in this group.

A large multi-institutional experience from a G erman registry between 1994 and 2002 has been presented in abstract form [8]. $\mathrm{H}$ artmann et al. collected data from 198 patients and classified them into three prognostic groups, similar to Bokemeyer. Patients in group A were initially diagnosed with metastatic GCT, including CN S metastases, and showed $O S$ at 2 years of $57 \%$. For patients in group B with isolated intra-cerebral relapse, os was $44 \%$, whilst for those in group $C$ with extra- and intracerebral recurrence of GCT, OS was only $26 \%$. In contrast to the study by Fossa et al., treatment with radiotherapy (and/or neurosurgery) was associated with better prognosis in those with brain metastastases at initial diagnosis, whilst in groups B and C local treatment alone with radiotherapy or neurosurgery was associated with poorer prognosis.

In keeping with the above series, investigators from Indiana University also found improved prognosis in patients presenting with brain metastases from testicular cancer at initial diagnosis or with isolated CNS relapse following complete response, compared with those who developed brain metastases at the time of systemic relapse [9]. A total of nine out of ten patients presenting with brain metastases were advanced stage III (one stage I) and all were treated with chemotherapy (with PVB + - - doxorubicin) plus whole-brain radiotherapy (WBRT). A total of three patients went into lasting remission. Of the four patients who developed a relapse confined to the brain after initial successful PVB therapy, three were treated with surgery and all had WBRT and platinum-containing chemotherapy. Three out of four patients achieved long-term survival. The group of ten patients who developed progressive disease and brain metastases after initial PVB, or those with brain metastases at presentation who did not respond to first-line systemic 
chemotherapy were all treated with WBRT. Median survival was 10 months, with four dying of systemic disease during or within 1 month of completing radiotherapy. A further four out of ten achieved good palliation of their brain metastases, but, overall, there were no long-term survivors.

The use of first-line high-dose chemotherapy in patients with brain metastases from advanced GCTs has been evaluated in 22 patients [10]. All patients received high-dose VIP (etoposide, ifosfamide and cisplatin), followed by autologous transplantation. Three patients al so had surgery. Brain irradiation was applied in 12 patients with symptomatic C N S disease or as consolidation in case of residual brain lesions after chemotherapy. There were two deaths (one owing to brain hemorrhage, and the other owing to sepsis). A total of two out of the nine patients treated with highdose chemotherapy alone relapsed both systemically and in the brain, while of the ten patients treated with both chemotherapy and radiotherapy alone, three recurred systemically and none in the brain. $0 f$ the remaining 20 patients, 2 -year progression-free and overall survival rates were 72 and $81 \%$, respectively.

'Looking back over this data, there emerges a trend towards better outc ome in patients who initia lly present with brain disease versus those who develop progression in the brain during or after systemic chemothera py, and those with a single bra in metastasis.'

In terms of radiotherapy dosage and timing, the situation is again unclear. In the German series, radiotherapy was given as WBRT of 30-45 Gy over 20-25 fractions. In patients with isolated lesions, an additive 10-Gy boost was given. $N$ one of the five patients in the series given single-modality radiotherapy treatment survived over 2 months. The authors recommended that radiotherapy be used as palliation in those who develop brain metastasis during systemic treatment as sustained survival will not be reached. In the Indiana series, in patients with brain metastases at initial diagnosis, radiotherapy was given concomitantly with chemotherapy to the whole brain as $50 \mathrm{~Gy}$ in 25 fractions. Patients with solitary relapse confined to the brain had surgical excision followed by WBRT given as 45-50 Gy in 22-25 fractions. In the updated Indiana series by Spears et al., those patients who were alive and well after WBRT had not developed any significant late complications detectable by history and physical examination. In contrast to the above, Fossa et al. found radiotherapy, given in a similar method, had no impact on $O S$ in patients who had brain metastases at presentation. Hartmann et al. have found an impact of radiotherapy; however, details of the radiotherapy and chemotherapy treatments are awaited.

The role of neurosurgery is also contentious. In the Indiana series none of the long-term survivors with brain metastases at presentation had neurosurgery, but in those with previous complete response to chemotherapy and solitary brain metastasis on relapse, three out of five patients had long-term survival with surgery followed by chemotherapy and radiotherapy. C rabb et al. describe the brain as a sanctuary site for relapse in patients with isolated relapse occurring after successful chemotherapy for metastatic GCT [11]. Six patients in their series fall into this category and all of these patients had an aggressive salvage approach with neurosurgery followed by radiotherapy. Initially, they all went into remission and there were four long-term survivors. In their series they conclude that there is no role in further chemotherapy as adjunct or prophylaxis against further systemic relapse.

In terms of prognostic features, both the Bockemeyer and Fossa series found, upon univariate analysis, a more favorable outcome of patients with single as opposed to multiple brain metastases at initial staging. Bockemeyer et al. also observed that the extent and sites of additional metastatic disease did not significantly influence long-term survival (although nearly $90 \%$ of patients with brain metastases were al so found to have lung spread). Both the elevation of human chorionic gonadatrophin and the histology of the primary testis cancer were not of significant influence on long-term outcome. H owever, contrary to this, there is some unpublished data suggesting that the presence of metastatic choriocarcinoma indicates a poor prognosis, independent from any form of treatment.

Looking back over this data, there emerges a trend towards better outcome in patients who initially present with brain disease versus those who develop progression in the brain during or after systemic chemotherapy, and those with a single brain metastasis. In terms of chemotherapy regime, there is perhaps some rather sketchy evidence pointing towards a more favorable outcome with more dose-intense or specialist CNS protocols, though the numbers in 
the series are small. Evidence regarding the optimal sequence of treatment modalities and indeed the whole role of multimodality treatment with radiotherapy and neurosurgery is equivocal. Furthermore, the prognosis of patients who have relapsed in the brain during or after chemotherapy for systemic metastases is particularly poor. Is there a role of CN S prophylaxis in patients who are thought to be at high risk of developing brain metastases, as is the case with, for example, lymphomas and gestational trophoblastic tumors? To this end, prognostic factors need to be defined.

'The rela tive rarity of this c linic al setting makes large clinic al tria ls unfeasible to help define best practice. However, there clearly needs to be better pooling and retrospective study of our collective experience.'

There clearly remains uncertainty as to the management of brain metastases from GCTs. This was highlighted by a survey of UK Cancer Centers in 2001. It was found that there were significant variations in treatment with different systemic chemotherapy regimes employed (BEP was the most commonly used treatment, in $36 \%$ of sites). Intrathecal or high-dose intravenous methotrexate was rarely used. The practice of radiotherapy and neurosurgery also varied considerably. It was evident that only around half of the centers actually had specific guidelines as to the management of these patients (unpublished data). The relative rarity of this clinical setting makes large clinical trials unfeasible to help define best practice. H owever, there clearly needs to be better pooling and retrospective study of our collective experience. O nly then will we be able to better define prognostic parameters and answer the fundamental issues that exist. We strongly believe that patients with poor-prognosis GCTs should be managed in selected specialized cancer centers and that we must try and develop optimized protocols for complex cases, including the treatment of brain metastases from GCTs.

Financial \& competing interests disclosure The authors have no relevant affiliations or financial involve ment with any organization or entity with a financial interest in or financial conflict with the subject matter or materials discussed in the manuscript. This includes employment, consultancies, honoraria, stock ownership or options, expert testimony, grants or patents received or pending, or royalties.

$\mathrm{N}$ o writing assistance was utilized in the production of this manuscript.

\section{Bibliography}

1. International G erm Cell Consensus Classification: a prognostic factor-based staging system for metastatic germ cell cancers. International G erm Cell C ancer Collaborative Group. J. Clin. O ncol. 15(2), 594-603 (1997).

2. Williams SD, Einhorn LH : Brain metastases in disseminated germinal neoplasms: incidence and clinical course. C ancer 44(4), 1514-1516 (1979).

3. Rustin GJ, N ewlands ES, Bagshawe KD et al.: Successful management of metastatic and primary germ cell tumors in the brain. Cancer 57(11), 2108-2113 (1986).

4. Brindley $C J$, Antoniw $P, N$ ewlands ES et al.: Pharmacokinetics and toxicity of the epipodophyllotoxin derivative etoposide (VP 16-213) in patients with gestational choriocarcinoma and malignant teratoma. Cancer Chemother. Pharmacol. 15(1), 66-71 (1985).

5. Ginsberg S, Kirshner J, Reich S et al.: Systemic chemotherapy for a primary germ cell tumor of the brain: a pharmacokinetic study. Cancer Treat. Rep. 65(5-6), 477-483 (1981).
6. Bokemeyer $\mathrm{C}, \mathrm{N}$ owak $\mathrm{P}, \mathrm{H}$ aupt $\mathrm{A}$ et al.: Treatment of brain metastases in patients with testicular cancer. J. Clin. O ncol. 15(4), 1449-1454 (1997).

7. Fossa SD, Bokemeyer C, Gerl A et al.: Treatment outcome of patients with brain metastases from malignant germ cell tumors. Cancer 85(4), 988-997 (1999).

8. H artmann JT, Bamberg $M$, Albers $P$ et al.: $M$ ultidisciplinary treatment and prognosis of patients with central nervous metastases (CNS) from testicular germ cell tumor (GCT) origin. Proc. Am. Soc. Clin. O ncol. 22, 400 (2003) (Abstract 1607).

9. Spears WT, M orphis JG 2nd, Lester SG et al.: Brain metastases and testicular tumors: long-term survival. Int. J. Radiat. Oncol. Biol. Phys. 22(1), 17-22 (1992).

10. Kollmannsberger $\mathrm{C}, \mathrm{N}$ ichols $\mathrm{C}, \mathrm{Bamberg} \mathrm{M}$ et al.: First-line high-dose chemotherapy $+/$ radiation therapy in patients with metastatic germ-cell cancer and brain metastases. Ann. Oncol. 11(5), 553-559 (2000).

11. Crabb SJ, M cKendrick JJ, M ead GM : Brain as sanctuary site of relapse in germ cell cancer patients previously treated with chemotherapy. Clin. O ncol. (R. Coll. Radiol.) 14(4), 287-293 (2002).
Affiliations

- Sanjay Raj Box 193, Oncology Centre, Addenbrookés $\mathrm{H}$ ospital, Hills Road, Cambridge CB2 OQ Q, UK sanjay.raj@addenbrookes.nhs.uk

- Christine Parkinson Box 193, Oncology Centre, Addenbrookés H ospital, Hills Road, Cambridge CB2 0Q Q, UK christine.parkinson@addenbrookes.nhs.uk

- Michael Williams Box 193, Oncology Centre, Addenbrookés H ospital, Hills Road, Cambridge CB2 OQ Q, UK michael.williams@addenbrookes.nhs.uk

- Danish M azhar Box 193, Oncology Centre, Addenbrookés H ospital, Hills Road, Cambridge CB2 OQ Q, UK danish.mazhar@addenbrookes.nhs.uk 